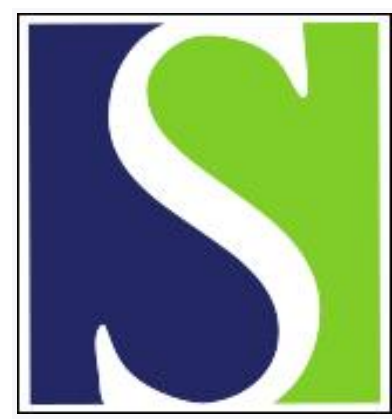

Scand J Work Environ Health 2006;32(1):67-74

https://doi.org/10.5271/sjweh.978

Issue date: 31 Feb 2006

Predicting the duration of sickness absence for patients with common mental disorders in occupational health care

by Nieuwenhuijsen K, Verbeek JHAM, de Boer AGEM, Blonk RWB, van Dijk FJH

Affiliation: Coronel Institute of Occupational Health, Academic Medical Centre, University of Amsterdam, PO Box 22700, 1100 DD Amsterdam, The Netherlands. K.Nieuwenhuijsen@amc.uva.nl

The following articles refer to this text: 2008;34(1):23-32;

2010;36(6):515-516; 2011;37(5):359-362; 2012;38(2):93-104;

2013;39(2):144-154; 2014;40(2):195-202; 2018;44(3):274-282;

2020;46(2):168-176; 2021;47(6):435-445; 2022;48(7):579-585

Key terms: mental disorder; mental health problems; occupational health care; prediction rule; prognosis; prospective cohort; return to work; sickness absence

This article in PubMed: www.ncbi.nlm.nih.gov/pubmed/16539174 


\title{
Predicting the duration of sickness absence for patients with common mental disorders in occupational health care
}

\author{
by Karen Nieuwenhuijsen, PhD, ${ }^{1}$ Jos HAM Verbeek, PhD, ${ }^{1}$ Angela GEM de Boer, PhD, ${ }^{1}$ \\ Roland WB Blonk, PhD, ${ }^{2}$ Frank JH van Dijk, PhD ${ }^{1}$
}

\begin{abstract}
Nieuwenhuijsen K, Verbeek JHAM, de Boer AGEM, Blonk RWB, van Dijk FJH. Predicting the duration of sickness absence for patients with common mental disorders in occupational health care. Scand $\mathrm{J}$ Work Environ Health 2006;32(1):67-74.
\end{abstract}

\begin{abstract}
Objectives This study attempted to determine the factors that best predict the duration of absence from work among employees with common mental disorders.

Methods A cohort of 188 employees, of whom 102 were teachers, on sick leave with common mental disorders was followed for 1 year. Only information potentially available to the occupational physician during a first consultation was included in the predictive model. The predictive power of the variables was tested using Cox's regression analysis with a stepwise backward selection procedure. The hazard ratios (HR) from the final model were used to deduce a simple prediction rule. The resulting prognostic scores were then used to predict the probability of not returning to work after 3, 6, and 12 months. Calculating the area under the curve from the ROC (receiver operating characteristic) curve tested the discriminative ability of the prediction rule.

Results The final Cox's regression model produced the following four predictors of a longer time until return to work: age older than 50 years [HR $0.5,95 \%$ confidence interval $(95 \% \mathrm{CI}) 0.3-0.8$ ], expectation of duration absence longer than 3 months (HR 0.5, 95\% CI 0.3-0.8), higher educational level (HR 0.5, 95\% CI 0.3-0.8), and diagnosis depression or anxiety disorder (HR $0.7,95 \%$ CI $0.4-0.9$ ). The resulting prognostic score yielded areas under the curves ranging from 0.68 to 0.73 , which represent acceptable discrimination of the rule.

Conclusions A prediction rule based on four simple variables can be used by occupational physicians to identify unfavorable cases and to predict the duration of sickness absence.
\end{abstract}

Key terms mental health problems; prediction rule; prognosis; prospective cohort; return to work.

Mental disorders such as adjustment disorder, depression, and anxiety disorder occur frequently and are often disabling. Loss of work productivity and sickness absence are two of the negative consequences of these common mental disorders. In the United Kingdom, common mental disorders were found to be the second major cause of sickness absences longer than 21 days (1)

An early estimate of the prognosis for patients with common mental disorders could serve as a point of departure for both the identification of cases at risk for long-term absence and as an instrument for predicting the course of the disorder. Whereas this use applies to all areas of health care, the need for accurate prognoses is even more pronounced in occupational health care. For physicians who are involved in the rehabilitation and management of return to work, giving correct advice about prognosis is an important aspect of their activities.

A recent study on common mental disorders in primary care identified the following major predictors of slow symptom recovery: severity of the disorder, low educational level, and a long pre-baseline duration of the episode (2). However, a restoration of work functioning does not always follow symptom recovery in common mental disorders (3-5). This contradiction agrees with the assumptions of the International Classification of Functioning, Disability and Health (ICF) model (6). This model acknowledges that environmental factors, such as work characteristics, and personal factors, such as demographic and motivational factors,

2 TNO Work and Employment, Hoofddorp, The Netherlands.

Correspondence to: Dr Karen Nieuwenhuijsen, Coronel Institute of Occupational Health, Academic Medical Centre, University of Amsterdam, PO Box 22700, 1100 DD Amsterdam, The Netherlands. [E-mail: K.Nieuwenhuijsen@amc.uva.nl] 
may influence health. Therefore, not only do disorderrelated factors need to be considered as possible predictors of return to work, but also environmental and personal factors should be considered.

In this study, in order to maximize practical value, we have taken into account only predictive factors that are potentially available to the occupational health physician during the first consultations. Consequently, information that would require more effort than interviewing the patient or administering a simple questionnaire is disregarded. To our knowledge, no comprehensive review on factors that influence return to work among employees with common mental disorders has been published. Therefore, we reviewed the literature in search of disorder-related, personal, and environmental predictor variables that fulfilled our criterion of practical value in studies on either recovery from work disability among patients with common mental disorders (7-9) or duration of sickness absence in general (10-22).

With regard to disorder-related variables, it was found that a mental health problem, as opposed to a physical health problem, is related to a longer duration of sickness absence among employees on sick leave (19). Within a group of employees with mental health problems, the length of sickness absence was found to vary according to the diagnostic category (21). Furthermore, a higher level of depressive symptoms was related to a longer duration of the sickness absence $(7,18)$. Employees who considered their absence due to mental health problems to be work-related stayed off work longer (9). Employees who have been absent for a longer period of time (20) (number of pre-baseline days) or have been experiencing symptoms for a longer time are at risk of a longer duration of sickness absence (8). Being a female increased the likelihood of return to work in two studies $(8,21)$, while it decreased this likelihood in another study (10). Older age was predictive of not returning to work $(10,13,17-18,21-22)$. Being divorced also decreased the likelihood of return to work (10). A higher educational level increased the chance of return to work $(18,22)$. Positive recovery expectations were associated with a shorter duration of the sickness absence (11-12). High job demands is an environmental factor that is predictive of prolonged sickness absence (15-16). Low supervisory and co-worker support was found to be related to a longer duration of sickness absence (14-16).

On the basis of the aforementioned findings from the literature, we included the following predictors in this study: (i) disorder-related factors (diagnosis, level of depressive symptoms, work-relatedness of the disorder, pre-baseline duration of the disorder, pre-baseline sick leave days), (i) personal factors (gender, age, marital status, recovery expectations, educational level), and (iii) environmental factors (job demands, supervisory support, co-worker support). Accordingly, our research question was "Which of these disorder-related, personal, and environmental factors are predictive of the duration of sickness absence among employees with common mental disorders?"

\section{Study population and methods}

\section{Participants and procedure}

As part of a longitudinal cohort study of employees with mental health problems, 30 occupational health physicians from nine occupational health services provided data on consecutive patients. Because we wanted only patients in the same stage of disease, an inception cohort, eligible employees had to have been on full sick leave for less than 6 weeks due to mental health problems. Mental health problems were defined as psychological symptoms that were not caused by a somatic disorder. Any previous consultation with the occupational physician had to be more than 3 months earlier. The occupational health physicians reported 277 employees as being eligible for participation. Of these patients, 66 (24\%) refused to participate. Ultimately, 198 employees filled out the baseline questionnaire. For the purpose of this study, only data from participants with common mental disorders were used $(\mathrm{N}=188)$.

Each participant was interviewed by the researchers by telephone. Subsequently, four questionnaires were sent to the participants by mail at baseline $\left(\mathrm{t}_{0}\right), 3$ months $\left(\mathrm{t}_{1}\right), 6$ months $\left(\mathrm{t}_{2}\right)$, and 12 months $\left(\mathrm{t}_{3}\right)$. One reminder was sent to each participant who did not return the questionnaire within 2 weeks.

\section{Measures}

\section{Diagnostic interview}

The participants were diagnosed by means of a telephone version of the structured Composite International Diagnostic Interview (CIDI) (23), which included the following diagnostic categories: major depressive disorder, panic disorder, social phobia, somatoform disorder, bipolar disorder, obsessive-compulsive disorder, post-traumatic stress disorder, and psychotic disorder. For the first three diagnoses, we administered the full CIDI scales, while we used the screen questions only for the other categories. Common mental disorder was operationalized as meeting the criteria for depression (major depressive disorder), anxiety disorder (panic disorder, social phobia, somatoform disorder, obsessivecompulsive disorder, or post-traumatic stress disorder), or adjustment disorder. The patients with the last diagnosis were defined as being on sick leave due 
to psychological symptoms, but without meeting the criteria for one of the other disorders.

\section{Outcome}

Data on return to work were collected by means of repeated questionnaires. If inconsistencies were discovered or if absence data could not be retrieved from the questionnaire, the sick leave register of the occupational health service was consulted. Duration of sickness absence was measured by calculating the time to full return to work during the follow-up period, regardless of the timing of any partial return to work. Full return to work was operationalized as working the same number of hours as prior to the sickness absence episode, for at least 1 week. Relapses after 1 week were not accounted for. The follow-up period could exceed 365 days due to the time between the first day of absence and the time of baseline measurement.

\section{Predictors}

Disorder-related factors. We selected the following disorder-related factors from the literature: diagnosis (adjustment disorders versus depressive or anxiety disorders according to the interview), level of depressive symptoms [DASS-depression (24), using the cut-off point of $>12$ (25)], work-relatedness of the disorder (self-report, single item "What, in your opinion, is the main reason for your current sickness absence?"; answers were dichotomized into work-related versus not work-related), the pre-baseline duration of the disorder ( $<3$ months versus $\geq 3$ months). Pre-baseline sick leave days were defined as the self-reported number of days on sick leave in the year preceding the current episode of sick leave ( 0 days versus $\geq 1$ day). Diagnosis was dichotomized into adjustment disorder and depressive or anxiety disorder, because the latter two are considered more severe (26).

Personal factors. Personal factors included as potential predictors were gender (male, female), age ( $<50$ years, $\geq 50$ years), marital status (married or living together, single, widowed or divorced), recovery expectations (self-report, single item "How many months do you think it will take you to fully return to work?"; answers were dichotomized into expected duration $\leq 3$ months versus $>3$ months), and educational level (low versus medium and high). Educational level was estimated on the basis of job title using a standard classification of occupations (27). Low educational level included primary school, lower vocational education, and lower secondary school. Medium or high educational level included intermediate vocational education, upper secondary school, upper vocational education, and university.
Environmental factors. The included environmental factors were job demands ("I have to work very hard"), supervisory support ("I can count on my supervisor whenever I encounter difficulties in my work"), and coworker support ("I can count on my colleagues whenever I encounter difficulties in my work"). Perceived job demands, supervisory support, and co-worker support were assessed with one-item questions using four-point Likert scales (range 1 "I strongly disagree" to 4 "I strongly agree").

\section{Statistical analysis}

First, we constructed a linear regression model of the duration of sickness absence in order to test the collinearity of the variables.

To establish predictors of the duration of sickness absence, a Cox's regression analysis was conducted. A backward stepwise procedure was used to identify relevant predictors. The elimination of nonsignificant predictors was based upon the Wald statistic $(<0.05)$, the factor with the highest P-value being removed first. Thereafter, the $-2 \log$ likelihood ratio test was used to assess whether this removal led to a significant decrease in the predictive power of the model. If not, then the predictor was removed from the model. The proportional hazards assumption of the predictors in the final Cox's regression model was tested by visual inspection of the $\log$ minus log graphs. We estimated survivor functions at 3, 6, 9, and 12 months for two hypothetical workers using the following equation:

$$
\mathrm{S}(\mathrm{t}, \mathrm{z})=\left(\mathrm{S}_{0}(\mathrm{t})\right)^{\exp (\beta 1 \mathrm{z} 1+\ldots+\beta 4 \mathrm{z} 4)},
$$

where $S_{0}(t)$ is the baseline survivor function, $\beta_{1} \ldots \beta_{4}$ are the regression coefficients estimated by the model, and $\mathrm{z}_{1} \ldots \mathrm{z}_{4}$ represent the score for each predictor ( 0 or 1$)$.

Because the formula is too complicated for swift use by clinicians, the beta coefficients from the final proportional hazards model were used to construct a simple clinical prediction rule. Therefore, we transformed the regression equation by assigning a score to each predictor in proportion to the magnitude of the beta coefficients from the equation. The scores of the four predictors were then added to a prognostic sum score.

In order to test its discriminative ability, we then used this simple sum score to predict the probability of still being on sick leave at 3, 6, and 12 months. From the ROC (receiver operating characteristic) curve, the "area under the curve" (AUC) was calculated to evaluate the discrimination of the prediction rule (28).

The internal validity of the prediction rule was examined by comparing the standard errors of the betas of the original survival analysis with those of a bootstrap sample, which was drawn with replacement from the original sample. The averages of the performance measures were taken over 1000 repetitions. 


\section{Results}

\section{Participant characteristics}

Two of the 188 original participants did not return any of the follow-up questionnaires (lost to follow-up). Overall, 53 (7\%) of the four questionnaires sent to each of the remaining 186 participants were missing. A nonresponse analysis revealed no statistically significant differences for all but one possible predictor and none for outcome. Compared with the participants without missing questionnaires, those with one or more questionnaires missing more often had a low level of education $\left(30 \%\right.$ versus $\left.18 \%, \chi^{2}=3.9, \mathrm{P}<0.05\right)$. Table 1 presents the baseline value of the potential predictors for the employees. The duration of sickness absence ranged from 7 to 476 days. At the end of the follow-up, 133 $(71 \%)$ employees had fully returned to work.

\section{Prediction model}

The smallest eigenvalue in the collinearity diagnostic was 0.16 , whereas an eigenvalue of $<0.10$ suggests collinearity (29). A visual inspection of the log minus log graphs gave no indication of a violation of the proportional hazard assumption. Table 2 presents the variables that were retained in the final Cox's regression model

Table 1. Number and percentage or mean and standard deviation (SD) of potential predictors of time until return to work due to common mental disorders; due to missing values, the number (N) ranges from 170 to 186 .

\begin{tabular}{|c|c|c|c|c|}
\hline Potential predictor & $\mathrm{N}$ & $\%$ & Mean & SD \\
\hline \multicolumn{5}{|l|}{ Disorder-related factors } \\
\hline Diagnosis, anxiety disorder or depression & 66 & 36 & . & . \\
\hline $\begin{array}{l}\text { Severity of depressive symptoms, cut- } \\
\text { off score } 12\end{array}$ & 118 & 63 & . & . \\
\hline $\begin{array}{l}\text { Cause of common mental disorder, } \\
\text { work-related }\end{array}$ & 125 & 67 & . & . \\
\hline $\begin{array}{l}\text { Pre-baseline duration of symptoms, } \\
\geq 3 \text { months }\end{array}$ & 144 & 78 & . & . \\
\hline $\begin{array}{l}\text { Pre-baseline days of sickness absence, } \\
>0 \text { days in previous year }\end{array}$ & 122 & 67 & . & . \\
\hline \multicolumn{5}{|l|}{ Personal factors } \\
\hline Age, $\geq 50$ years & 56 & 30 & . & . \\
\hline Gender, male & 74 & 40 & . & . \\
\hline \multicolumn{5}{|l|}{ Marital status } \\
\hline Married or living together & 147 & 80 & . & . \\
\hline Single & 20 & 11 & . & . \\
\hline Divorced or widowed & 16 & 9 & . & 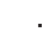 \\
\hline Educational level, medium or high & 146 & 80 & · & 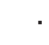 \\
\hline Recovery expectation, duration $>3$ months & 45 & 26 & . & 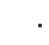 \\
\hline \multicolumn{5}{|l|}{ Environmental factors } \\
\hline Job demands & . & . & 2.8 & 0.9 \\
\hline Supervisory support & . & . & 2.4 & 0.9 \\
\hline Co-worker support & . & . & 3.0 & 0.7 \\
\hline
\end{tabular}

a Employees working in the education sector did not statistically differ from other workers with respect to this variable ( $59 \%$ versus $46 \%$; $\chi^{2}=1.05, P>0.3$ ) after the backward elimination process. This process yielded the following four statistically significant predictors of a longer time to return to work: age $\geq 50$ years, patient expectation of duration absence $>3$ months, education level medium or high, and diagnosis depression or anxiety disorder.

As can be seen from table 3, patient A had substantially higher probabilities of not returning to work at 3 , 6,9 , and 12 months than patient B.

The following factors were not predictive of the duration of sickness absence [hazard ratios (HR) of the full model are presented): level of depressive symptoms (HR 0.7 ), work-relatedness of the disorder (HR 1.0), prebaseline duration of the symptoms (HR 0.7), pre-baseline days of sickness absence (HR 1.1), gender (HR 0.9), marital status (HR 0.7), job demands (HR 1.0), supervisory support (HR 1.1), and co-worker support (HR $0.9)$. Post-hoc analyses were performed to (i) examine the possibility of the educational level effect being an effect of occupation (teacher versus nonteacher) and (ii) examine the impact of later interventions by the occupational physician (good care versus poor quality care). Good care was operationalized as adherence to the Dutch practice guidelines (30), as assessed according to 11 performance indicators (31). The sum score of these indicators was dichotomized by applying a cutoff point

Table 2. Final model of the stepwise backward Cox's regression of predictors of the duration of sickness absence $(\mathrm{N}=168$ due to missing cases in predictors). This table only presents results with a P-value of $<0.05$. ( $95 \% \mathrm{Cl}=95 \%$ confidence interval)

\begin{tabular}{lccc}
\hline Predictor & Beta & $\begin{array}{c}\text { Hazard } \\
\text { ratio }^{\text {a }}\end{array}$ & $95 \% \mathrm{Cl}$ \\
\hline Age, $\geq 50$ years & -0.70 & 0.5 & $0.3-0.8$ \\
Patient's recovery expectation, & -0.71 & 0.5 & $0.3-0.8$ \\
duration $>3$ months & & & $0.3-0.8$ \\
Educational level, medium or high & -0.72 & 0.5 & 0.3 \\
Diagnosis, anxiety disorder or depression & -0.42 & 0.7 & $0.4-0.9$ \\
\hline
\end{tabular}

a $A$ hazard ratio of $<1$ indicates the risk of a longer time to return to work compared with the reference group of each predictor (age $<50$ years, recovery expectation $\leq 3$ months, lower educational level, diagnosis adjustment disorder).

Table 3. Survivor functions and estimated probabilities of not returning to work on the basis of the final Cox's regression model for two hypothetical patients ( $N=168$ due to missing cases for the predictors).

\begin{tabular}{lccc}
\hline Time & Survivor function & ${\text { Patient } \mathrm{A}^{\mathrm{a}}}$ & Patient $^{\mathrm{b}}$ \\
\hline 3 months & 0.88 & 0.97 & 0.64 \\
6 months & 0.63 & 0.89 & 0.23 \\
9 months & 0.48 & 0.83 & 0.10 \\
12 months & 0.32 & 0.76 & 0.03 \\
\hline
\end{tabular}

a Patient $A$ is older than 50 years, expects to be off work longer than 3 months, and has a high educational level and a depressive disorder.

b Patient $B$ is younger than 50 years, expects to return to work within 3 months, has a lower education, and suffers from an adjustment disorder. 
based upon its median score. Both post-hoc analyses were conducted by adding this factor to the final Cox's regression model. It was found that being a teacher was not predictive of the duration of sickness absence (HR 1.3), nor did the addition of this factor change the statistical significance of the predictors of the original model. Good care by the occupational health physician was predictive of the duration of sickness absence (HR 0.6 ), and its addition led to the predictor diagnosis becoming marginally statistically significant $(\mathrm{P}=0.07)$.

\section{Clinical prediction rule}

The magnitude of the beta coefficients of the four predictive factors was of the same order. This outcome enabled us to compute the sum score by simply adding one point for each predictive factor present. The clinical prediction rule stated that one point should be assigned for each of the following employee characteristics: being older than 50 years, expecting to be off work longer than 3 months, having a middle or high educational level, and having either a depressive or anxiety disorder. Consequently, the prognostic sum score ranged from 0 (no predictors with a score of 1 to 4 (four predictors with a score of 1). Figure 1 shows the survival curve for the number of days of sickness absence with separate lines for employees with different prognostic sum scores. A prognostic score of 4 has a slightly lower number of days of sickness absence, but the group was very small $(\mathrm{N}=8)$.

The discriminative ability of the prognostic sum score was found to be acceptable (32). Therefore, with the use of the sum score, the probability of returning to work can be fairly well predicted. This outcome can be considered as an indication of the fit of the model to the data. The ROC analysis revealed that the AUC for the rate of return to work after 6 months was 0.71 [95\% confidence interval (95\% CI 0.63-0.78)], while it was 0.73 (95\% CI 0.65-0.81) after 12 months and 0.68 (95\% CI 0.59-0.77) after 3 months. An additional ROC analysis of the sum score using the magnitude of the beta coefficients as weights did not enhance the discriminative ability (eg, the AUC after 12 months was 0.74 when compared with 0.73 ). For reasons of clinical feasibility it was therefore decided to maintain the simple sum score of one point for each predictor.
Table 4 shows that the differences between the standard error of the betas of the Cox's regression model in the original and the bootstrap sample (bias) were very small. This finding suggests that the standard errors from the original Cox's regression model were reliable, and, therefore, the internal validity of the sum score was sufficient. Thus, within this group of employees, the model seems stable.

\section{Discussion}

This study examined the factors predictive of a longer duration of sickness absence among employees with common mental disorders. Only age, recovery expectations, educational level, and diagnosis were found to predict the duration of sickness absence. These predictors of time to return to work differed from the major predictors of slow symptom recovery for common mental disorders. The hazard ratios from the multivariate model were utilized to devise a simple prediction rule,

\section{Survival function at prognostic sum score}

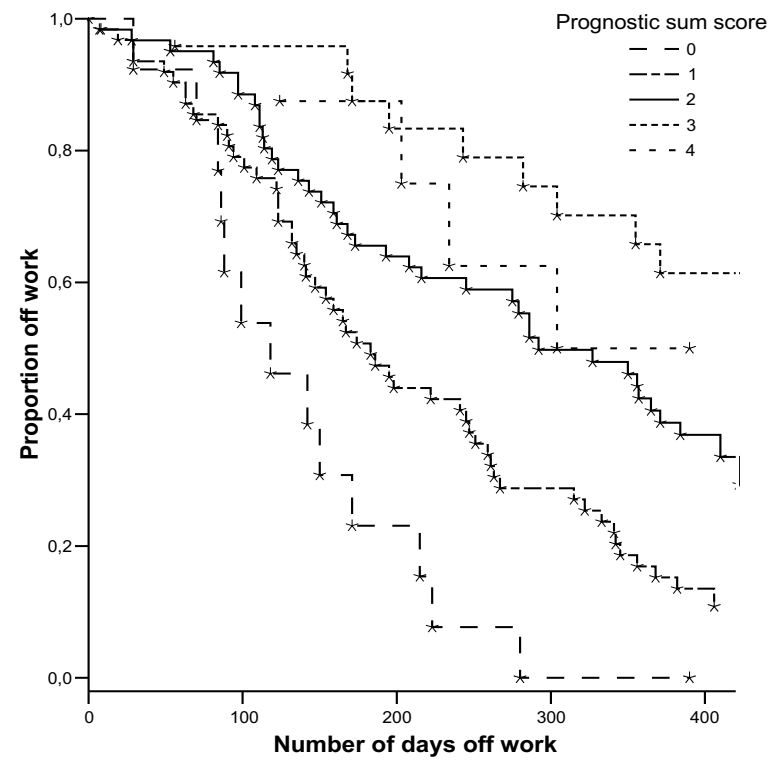

Figure 1. Number of days of sickness absence for the whole group ( $N=182$ due to missing values).

Table 4. Betas and standard errors (SE) of the predictors in the original and the bootstrap sample and also bias.

\begin{tabular}{|c|c|c|c|c|c|c|c|}
\hline \multirow[t]{2}{*}{ Predictor } & \multicolumn{2}{|c|}{ Original sample } & \multicolumn{2}{|c|}{ Bootstrap sample } & \multicolumn{2}{|c|}{$\begin{array}{c}\text { Beta in } \\
\text { bootstrap sample }\end{array}$} & \multirow[t]{2}{*}{$\begin{array}{c}\text { Bias (SE original - } \\
\text { SE bootstrap) }\end{array}$} \\
\hline & Beta & SE & Beta & SE & P5 & P95 & \\
\hline Age & -0.70 & 0.23 & -0.70 & 0.22 & -1.12 & -0.36 & 0.01 \\
\hline Patient's recovery expectation & -0.71 & 0.25 & -0.71 & 0.24 & -1.13 & -0.36 & 0.00 \\
\hline Educational level & -0.72 & 0.22 & -0.72 & 0.22 & -1.06 & -0.40 & -0.03 \\
\hline Diagnosis & -0.42 & 0.20 & -0.42 & 0.20 & -0.77 & -0.10 & -0.00 \\
\hline
\end{tabular}


which showed acceptable discrimination and internal validity.

The prospective design of this study has the advantage that all of the predictors were assessed at baseline, while outcome was measured during the follow-up. Consequently, the assessment of predictors was conducted without knowledge of outcome for either the employees or the researchers. Furthermore, the outcome event was clearly defined and clinically important, and the misclassification rate was tested by calculating the AUC. These aspects of our study meet the methodological criteria for prediction rules formulated by previous authors (33-34).

Ideally, the prediction rule should have been derived from one cohort of employees and tested on another to establish adequate external validity. Furthermore, even though our cohort comprised employees with diverse occupations, teachers constituted a relatively large proportion of the sample and resulted in an overrepresentation of employees with higher levels of education. However, even when the effect of profession was controlled in the post-hoc analyses, the predictors in the prediction rule maintained the prediction of the duration of sickness absence. With regard to the generalization of these results to more heterogeneous populations, caution is required even though common mental disorders are of particular concern within the teaching profession $(8,35)$.

Negative recovery expectations were found to be predictive factors of not returning to work in previous studies with employees with soft tissue injuries (11-12). Our study seems to corroborate these findings. One possible mechanism is that positive outcome expectancies represent the self-efficacy expectations of the employees. Self-efficacy in illness refers to one's confidence or belief that one can achieve a specific behavior despite one's illness (36-37). Return to work may be enhanced by a positive perception of the likelihood to return to work. On the other hand, employees may be best capable of predicting the duration of the sickness absence by taking into account their past experiences and their work and home environments. The factor "recovery expectations" was the only predictive factor in our model that was amendable to change, for instance, due to guidance by occupational health physicians. Occupational rehabilitation with a strong focus on altering negative outcome expectancies, for instance, by using cognitive-behavioral interventions, should be evaluated for its effectiveness on duration of sickness absence in an experimental design.

Among the predictive factors of the final model, the work-related variables were absent. This outcome may indicate that the duration of sickness absence is a reflection of coping with illness rather than coping with work conditions. Another possible explanation for the absence of work-related variables in the final model is that these types of work characteristics may be too complicated to be completely covered by one-item questions. We cannot exclude that a more elaborate assessment of work-related factors may lead to different results. A low educational level proved to be predictive of long-term sickness absence in a cohort of Danish employees (22). Surprisingly, we found that a high level of education was predictive of a longer time to return to work. This unexpected finding corresponds with the results of a recent study on work resumption among employees with adjustment disorders (38). One possible explanation may be that highly educated employees have more complex jobs, which may be especially hard to return to if one has developed a mental problem.

Our choice was to include only variables that were potentially available to an occupational health physician during a first consultation. Therefore, information on treatment or on efforts to enhance return to work by employers was disregarded. The confounding influence of treatment after the prognosis has been established during the first contact was examined in the post-hoc analyses. While three of the four predictors remained independent predictors of the duration of sickness absence, the influence of diagnosis appeared to be somewhat confounded by following treatment. Our restriction to information potentially available during a first consultation has practical value, but the additional benefit of elaborate treatment of employees with poor prognoses and the possible interaction between treatment and diagnosis still needs to be established in a randomized control trial.

We recommend that occupational health physicians assess information on the patient's expectation of the duration, together with the routinely gathered information on age, diagnosis, and educational level, to acquire prognostic information. The use of the prediction rule could then help identify potentially unfavorable cases. We further recommend that our prediction rule be validated in another population of employees with common mental disorders.

In conclusion, a simple prediction rule based on four baseline variables adequately predicts the duration of sickness absence for employees with common mental disorders. Older age, negative recovery expectations, a medium or high level of education, and being diagnosed with a depression or anxiety disorder are related to poor prognosis. Future prospective studies are needed to examine the prospective validity of this prediction rule.

\section{Acknowledgments}

Grants for this study were obtained from The Netherlands Organization for Scientific Research (NWO), 
Netherlands Concerted Research Action "Fatigue at Work", and the Foundation for Replacement and Occupational Health in Education (Stichting Vf/BGZ).

\section{References}

1. Stansfeld S, Feeney A, Head J, Canner R, North F, Marmot M. Sickness absence for psychiatric illness: the Whitehall II Study. Soc Sci Med. 1995;40:189-97.

2. Van den Brink RH, Ormel J, Tiemens BG, Smit A, Jenner JA, van der MK, et al. Predictability of the one-year course of depression and generalized anxiety in primary care. Gen Hosp Psychiatry. 2002;24:156-63.

3. Bijl RV, Ravelli A. Current and residual functional disability associated with psychopathology: findings from the Netherlands Mental Health Survey and Incidence Study (NEMESIS). Psychol Med. 2000;30:657-68.

4. Mintz J, Mintz LI, Arruda MJ, Hwang SS. Treatments of depression and the functional capacity to work. Arch Gen Psychiatry. 1992;49:761-8.

5. Van Der Klink JJ, Blonk RW, Schene AH, van Dijk FJ. Reducing long term sickness absence by an activating intervention in adjustment disorders: a cluster randomised controlled design. Occup Environ Med. 2003;60:429-37.

6. World Health Organization (WHO). International classification of functioning, disability and health (ICF). Geneva: WHO; 2001.

7. Judd LL, Akiskal HS, Zeller PJ, Paulus M, Leon AC, Maser JD, et al. Psychosocial disability during the long-term course of unipolar major depressive disorder. Arch Gen Psychiatry. 2000;57:375-80.

8. Young AE, Russell J. Demographic, psychometric, and case progression information as predictors of return-to-work in teachers undergoing occupational rehabilitation. J Occup Rehabil. 1995;5:219-34.

9. Houtman ILD, Schoemaker CG, Blatter BM, de Vroome EMM, Berg Rvd, Bijl RV. Psychische klachten, interventies en werkhervatting [Psychological complaints, interventions, and return to work]. Hoofddorp (the Netherlands): TNO Arbeid; 2002 .

10. Cheadle A, Franklin G, Wolfhagen C, Savarino J, Liu PY, Salley C, et al. Factors influencing the duration of workrelated disability: a population-based study of Washington State workers' compensation. Am J Public Health. 1994;84:190-6.

11. Cole DC, Mondloch MV, Hogg-Johnson S. Listening to injured workers: how recovery expectations predict outcomesa prospective study. Can Med Assoc J. 2002;166:749-54.

12. Hogg-Johnson S, Cole DC. Early prognostic factors for duration on temporary total benefits in the first year among workers with compensated occupational soft tissue injuries. Occup Environ Med. 2003;60:244-53.

13. Infante-Rivard C, Lortie M. Prognostic factors for return to work after a first compensated episode of back pain. Occup Environ Med. 1996;53:488-94.

14. Janssen N, van den Heuvel WP, Beurskens AJ, Nijhuis FJ, Schroer CA, van Eijk JT. The Demand-Control-Support model as a predictor of return to work. Int $\mathrm{J}$ Rehabil Res. 2003;26:1-9.

15. Krause N, Frank JW, Dasinger LK, Sullivan TJ, Sinclair SJ. Determinants of duration of disability and return-to-work af- ter work-related injury and illness: challenges for future research. Am J Ind Med. 2001;40:464-84.

16. Krause N, Dasinger LK, Deegan LJ, Rudolph L, Brand RJ. Psychosocial job factors and return-to-work after compensated low back injury: a disability phase-specific analysis. Am J Ind Med. 2001;40:374-92.

17. Reiso H, Nygard JF, Brage S, Gulbrandsen P, Tellnes G. Work ability and duration of certified sickness absence. Scand J Public Health. 2001;29:218-25.

18. Soderman E, Lisspers J, Sundin O. Depression as a predictor of return to work in patients with coronary artery disease. Soc Sci Med. 2003;56:193-202.

19. Hensing G, Spak F. Psychiatric disorders as a factor in sickleave due to other diagnoses: a general population-based study. Br J Psychiatry. 1998;172:250-6.

20. Alexopoulos EC, Burdorf A. Prognostic factors for respiratory sickness absence and return to work among blue collar workers and office personnel. Occup Environ Med. 2001;58:246-52.

21. Nystuen P, Hagen KB, Herrin J. Mental health problems as a cause of long-term sick leave in the Norwegian workforce. Scand J Public Health. 2001;29:175-82.

22. Eshoj P, Jepsen JR, Nielsen CV. Long-term sickness absence-risk indicators among occupationally active residents of a Danish county. Occup Med (Lond). 2001;51:347-53.

23. World Health Organization (WHO). Composite International Diagnostic Interview (CIDI), version 2.1. Geneva: WHO; 1997.

24. Lovibond SH, Lovibond PF. Manual for the depression anxiety stress scales (DASS). New South Wales (Australia): University of New South Wales; 1993.

25. Nieuwenhuijsen K, De Boer AG, Verbeek JH, Blonk RW, van Dijk FJ. The Depression Anxiety Stress Scales (DASS): detecting anxiety disorder and depression in employees absent from work because of mental health problems. Occup Environ Med. 2003;60 Suppl 1:I77-82.

26. Casey P, Dowrick C, Wilkinson G. Adjustment disorders: fault line in the psychiatric glossary. Br J Psychiary. 2001;179:479-81.

27. Netherlands Central Bureau of Statistics. Standaard Beroepen Classificatie [Standard Classification of Occupations]. The Hague: SDU/Publishers; 1993.

28. Hanley JA, McNeil BJ. The meaning and use of the area under a receiver operating characteristic (ROC) curve. Radiology. 1982;143:29-36.

29. Hocking RR. Methods and applications of linear models. New York (NY): Wiley; 1996.

30. Dutch Association for Occupational and Industrial Medicine (NVAB). Guidelines for the rehabilitation of employees with psychological disorders. Eindhoven (the Netherlands): NVAB; 2000.

31. Nieuwenhuijsen K, Verbeek JH, de Boer AG, Blonk RW, van Dijk FJ. Validation of performance indicators for rehabilitation of workers with mental health problems. Med Care. 2005;43(10): 1034-42.

32. Hosmer DW, Lemeshow S. Applied logistic regression. New York (NY): John Wiley \& Sons; 2000. p 160-4.

33. Laupacis A, Sekar N, Stiell IG. Clinical prediction rules: a review and suggested modifications of methodological standards. JAMA. 1997;277:488-94.

34. Wasson JH, Sox HC, Neff RK, Goldman L. Clinical prediction rules: applications and methodological standards. N Engl J Med. 1985;313:793-9.

35. Weber A, Weltle D, Lederer P. Zur Problematik krankheits- 
bedingter Frühpensionierungen von Gymnasiallehrkräften [IIlness related early pensioning of high school teachers]. Versicherungsmedizin 2002;54:75-83.

36. Bandura A. Self-efficacy: toward a unifying theory of behavioral change. Psychol Rev. 1977;84:191-215.

37. Lorig K, Gonzalez V. The integration of theory with practice: a 12-year case study. Health Educ Q. 1992;19:355-68.
38. Van der Klink JJ. Back in balance: the development and evaluation of an occupational health intervention for workrelated adjustment disorders. Amsterdam: University of Amsterdam, Academic Medical Center; 2000.

Received for publication: 17 March 2004 